\title{
Development and validation of a prognostic model of vertebral refracture after percutaneous kyphoplasty
}

Feng Yuan ( $\nabla$ xzmuyf@163.com )

\section{Research article}

Keywords: Percutaneous kyphoplasty, Prognostic model, Vertebral refracture, Last absolute shrinkage and selection operator regression

Posted Date: October 1st, 2021

DOI: https://doi.org/10.21203/rs.3.rs-871624/v1

License: (a) This work is licensed under a Creative Commons Attribution 4.0 International License.

Read Full License 


\section{Abstract}

Purpose: To investigate the factors influencing refracture after percutaneous kyphoplasty and to develop and validate a prognostic model.

Method: We retrospectively collected clinical data in 392 patients with osteoporotic vertebral compression fractures who underwent percutaneous kyphoplasty at the Affiliated Hospital of Xuzhou Medical University from 1 January 2018 to 1 January 2020.Predictors significantly associated with refracture after PKP were selected based on last absolute shrinkage and selection operator regression. Then a prognostic model were developed and internal validated using enhanced Bootstrap validation.

Results: Among the 392 patients who included in this study, there were 19 refracture after percutaneous kyphoplasty(4.8\%). Four factors were selected by least absolute shrinkage and selection operator regression for significant association with refracture after percutaneous kyphoplasty, including body mass index, bone mineral density, unilateral puncture, and bone cement leakage. After enhance Bootstrap validation, the bias-corrected curve of the model fitted well with the apparent curve, with the area under ROC curve of 0.931 and $95 \% \mathrm{Cl}$ of $(0.789,0.936)$.

Conclusion: The prognostic model developed based on four clinical profiles: body mass index, bone mineral density, unilateral puncture, and bone cement leakage can be used to identify those at most risk of refracture after percutaneous kyphoplasty.

\section{Introduction}

Percutaneous kyphoplasty (PKP) is a minimally invasive procedure for the treatment of osteoporotic vertebral compression fractures (OVCFs) [1,2]. It is performed primarily by injecting bone cement into vertebrae. Reinforcing the fractured vertebrae by injecting bone cement provides rapid pain relief and improves kyphosis. However, statistics show that the rate of new vertebral compression fractures in patients after PKP was approximately 8.7\%-18.4\% [3-6], which makes postoperative refracture an important issue for clinicians. Previous retrospective studies have reported several possible risk factors for refracture, including associations between age, body mass index (BMI), clinical complication rate, bone mineral density (BMD), number of initial surgical segments, and surgical puncture method [3, 6-13]. Despite years of research, there is no relevant literature that provides a valid prognostic model to predict the risk of refracture after PKP. Therefore, a prognostic model to predict the risk of refracture in patients after PKP would be of clinical value. This retrospective study analyzed the clinical data of refracture after PKP, and used the last absolute shrinkage and selection operator (LASSO) regression to select predictors of refracture after PKP.A prognosis model was then developed and validated to provide a reliable incidence of postoperative refracture. In order to identify people at high risk of refracture at an early stage and provide a scientific basis for subsequent clinical treatment.

This report follows the TRIPOD (Transparent Reporting of a Multivariate Prediction Rule for Individual Prognosis or Diagnosis) guidelines. Additional details are presented in the Supplementary Material. 


\section{Material And Methods}

Retrospective analysis of inpatient records from the Department of Spine Surgery, Xuzhou Medical University Hospital. We collected clinical data from 553 patients who were diagnosed with OVCFs and treated with percutaneous kyphoplasty between 1 January 2018 and 1 January 2020.All surgeries were performed by the same group of experienced and highly qualified chief surgeons. All patients were informed about the contents of the procedure and signed the Informed Consent Form for Surgery.

Inclusion criteria: $₫$ patients with thoracolumbar compression fractures caused by osteoporosis and fresh fractures within 2 weeks; $\nabla$ complete imaging data, no obvious intravertebral vacuum cleft and osteonecrosis manifestations on $\mathrm{CT}$, and obvious bone marrow edema signals visible on MRI; $₫$ patients without contraindications to surgery and with obvious indications for surgery; $\Downarrow$ postoperative follow-up time greater than 1 years and regular use of anti-osteoporosis drugs during the follow-up.

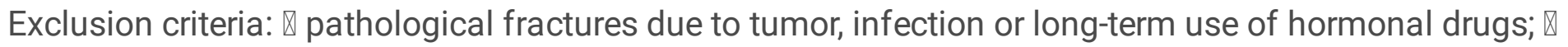
those with severe liver and kidney dysfunction, coagulation dysfunction or psychiatric disorders; $\nabla$ burst fractures due to a clear history of trauma to the first fracture and refracture. \#without undergone standard and scientific anti-osteoporosis treatment after surgery. Standard and scientific antio-steoporosis treatment was defined as regular oral calcium, vitamin $\mathrm{D}$ or use of zoledronic acid medication at least 1 time.

This study was approved by the Institutional Ethics Committee of the Affiliated Hospital of Xuzhou Medical University.

The basic information, surgical records, and follow-up of patients were retrospectively collected, including age, gender, general condition, history of cardiovascular disease, surgical segment, BMI, bone cement leakage, and surgical puncture method. BMI was measured by dual energy X-ray absorptiometry (Hologic Inc., Waltham, MA, USA) with preoperative lumbar spine (L1-L4) T values. Postoperative refractures were diagnosed mainly by clinical presentation and imaging data. Two prognostic outcomes were defined by imaging data and grouped according to the prognostic outcome. Patients who showed fresh bone edema on magnetic resonance imaging (MRI) were categorized as the "Refracture". Those who did not show clinical symptoms related to OVCFs and obvious signs of fracture on imaging were categorized as the "Non-refracture". If MRI was contraindicated or could not be performed, a bone scan was required to determine the presence of increased uptake compatible with recent fracture. The imaging data were evaluated separately by two orthopedic surgeons on the Picture Archiving and Communication Systems (PACS), and any differences in measurements were re-evaluated by the two surgeons to achieve agreement.

\section{Statistical analysis}

Analyses were performed using $\mathrm{R} \circledast$ (version 4.0.3). Descriptive and inferential statistics were calculated to describe the sample. Continous variables were expressed as means \pm standard deviation (SD) 
according to the distribution. The Kruskal-Wallis rank sum test was used for comparison between groups for continous variable. Categorical variables were shown as frequencies (percentages), and compared by $\chi 2$ test. $P<0.05$ was considered as significant.

LASSO regression is used to select variables by narrowing the regression coefficients. By making the sum of the mean (or likelihood function) of the squared residuals adding $\lambda$ times the absolute value of the regression coefficients minimal. LASSO regression can be used to filter variables by adjusting the penalty coefficient $\lambda$. The larger $\lambda$ is, the stronger the penalty of the model. Since it can avoid the problem of overfitting, it is often used to deal with data with multiple cointegration.

Among the many influential factors associated with refracture, we considered those that could be routinely and accurately assessed: age, sex, BMI, BMD, number of initial surgical segments, history of cardiovascular disease, and surgical puncture method. We selected the predictors to be included in the final model based on the LASSO regression analysis to build the column line graph model. After 1000 enhanced Bootsrap validations, the receiver operating characteristic curve (ROC) and $95 \% \mathrm{Cl}$ were calculated. Area under the receiver operating characteristic curve (AUC) and calibration plots (comparing the mean predicted risks with the observed risk) were used to calculate model performance.

\section{Results}

\section{Patient characteristics}

A total of 392 patients who underwent PKP surgery for OVCFs at our institution were included in this study. The mean age was 73.1 years. All patients were followed for 20 months on average (range, 14 months to 26 months). A total of 19 patients had refracture after PKP with an incidence of $4.8 \%$. A comparison of the clinical data and associated risk factors between two groups is shown in Table 1. 
Table 1

Compared of the clinical data and associated risk factors between two groups

\begin{tabular}{|c|c|c|c|c|c|c|}
\hline Variable & $\begin{array}{l}\text { Refracture } \\
(19 / 392)\end{array}$ & $\begin{array}{l}\text { Non- } \\
\text { refracture } \\
(373 / 392)\end{array}$ & $\begin{array}{l}\text { Odds } \\
\text { Ratio }\end{array}$ & $\begin{array}{l}\text { 95\%Confidence } \\
\text { Interval }\end{array}$ & $\chi^{2}$ & $\begin{array}{l}P \text { - } \\
\text { value }\end{array}$ \\
\hline Gender & & & & & 0.06 & 0.494 \\
\hline Female,n(\%) & $14(74)$ & $284(76)$ & - & & & \\
\hline Male,n(\%) & $5(26)$ & $89(24)$ & 1.05 & $0.25,3.90$ & & \\
\hline Age(years) & $\begin{array}{l}74.84 \pm \\
5.85\end{array}$ & $\begin{array}{l}73.09 \pm \\
8.73\end{array}$ & 0.98 & $0.92,1.05$ & 1.24 & 0.229 \\
\hline$\geq 65$ & 19 & 308 & & & & \\
\hline$<65$ & 0 & 65 & & & & \\
\hline $\mathrm{BMI}\left(\mathrm{kg} / \mathrm{m}^{2}\right)$ & $\begin{array}{l}19.55 \pm \\
1.56\end{array}$ & $\begin{array}{l}22.8 \pm \\
3.38\end{array}$ & 0.58 & $0.43,0.73$ & 19.771 & 0.000 \\
\hline$\geq 18.5$ & 15 & 330 & & & & \\
\hline$<18.5$ & 4 & 43 & & & & \\
\hline BMD & $\begin{array}{l}-4.29 \pm \\
0.53\end{array}$ & $\begin{array}{l}-3.42 \pm \\
0.76\end{array}$ & 0.11 & $0.04,0.26$ & 22.206 & 0.000 \\
\hline $\begin{array}{l}\text { Number of initial surgical } \\
\text { segments }\end{array}$ & & & 0.95 & $0.42,1.89$ & 0.352 & 0.553 \\
\hline $1, \mathrm{n}(\%)$ & $12(63)$ & 258 (69) & & & & \\
\hline $2, n(\%)$ & $4(21)$ & $77(21)$ & & & & \\
\hline$\geq 3, n(\%)$ & $3(16)$ & $38(10)$ & & & & \\
\hline \multicolumn{7}{|l|}{ Leakage } \\
\hline No,n(\%) & $3(16)$ & $183(49)$ & - & - & & \\
\hline Yes,n(\%) & $16(84)$ & $190(51)$ & 8.48 & $2.21,46.3$ & & \\
\hline Cardiovascular disease & & & & & 0.049 & 0.503 \\
\hline No,n(\%) & $9(47)$ & $167(45)$ & - & - & & \\
\hline Yes,n(\%) & $10(53)$ & $206(55)$ & 1.06 & $0.34,3.32$ & & \\
\hline Puncture method & & & & & 2.065 & 0.115 \\
\hline Bilateral,n(\%) & $16(84)$ & 256 (69) & - & - & & \\
\hline Unilateral,n(\%) & $3(16)$ & $117(31)$ & 0.27 & $0.04,1.08$ & & \\
\hline
\end{tabular}


Development of the prognostic model

Logistic models were used to estimate the coefficients for each risk factor in refracture. Refracture after PKP outcome was used as the dependent variable. Eight predictor variables, including age, gender, BMD, BMI, number of initial surgical segments, history of cardiovascular disease, surgical puncture method and the occurrence of bone cement leakage, were included in the LASSO regression model to select variables associated with refracture after PKP. When $\log (\lambda)$ was 0.0045 , it showed that the model obtained excellent performance with the least number of independent variables(Fig. 1). BMD, BMI, number of initial surgical segments, surgical puncture method were included in the prognostic model. The final model: Logit $(P)=0.5387+(2.1041 \times$ Leakage $)+(-0.5345 \times B M I)+(-2.2157 \times B M D)+(-1.3430 \times$ Unilateral $)$. Based on the LASSO results, a nomogram model was developed using $\mathrm{R}$ software to predict refracture after percutaneous kyphoplasty(Fig. 2).

Evaluation and internal validation of the prognostic model

Internal validation was performed using the enhanced Bootstrap validation. After 1000 Bootstrap validations, the prognostic model calibration curves were plotted. The curves show that the apparent curve of the model fits well with the bias-corrected curve, indicating that the prognostic model is in good agreement (Fig. 3). After 1000 Bootstrap resamplings, the prognostic model had an AUC of 0.931 and $95 \% \mathrm{Cl}$ of $(0.789,0.936)$ proving that the model discriminates well. (Fig. 3$)$

\section{Discussion}

PKP has become increasingly popular in the treatment of OVCFs since the first case of PKP was successfully treated. PKP not only quickly eliminates the severe pain that is difficult to relieve with bed rest, but also effectively reduces cement leakage due to the presence of the expandable balloon [1, 14]. After surgery, patients can recover vertebral height, improve the kyphosis and maintain the normal sequence of the spine in a short time. As an enhanced recovery after surgery (ERAS) technique, PKP allows patients to get out of bed early for functional exercises after surgery, reducing multiple complications caused by long-term bed rest and improving patients' quality of life. However, any new clinical technique is often accompanied by a series of complications. Common complications after PKP surgery include bone cement leakage, pulmonary embolism, refracture and increased postoperative back pain. Refracture have received a significant amount of attention in previous studies due to the increased pain of reoperation as well as the financial cost to the patient. Lee [1] found that PKP did not increase the risk of refracture in patients with OVCFs compared to conventional conservative treatment. PKP is not a major risk factor for vertebral fracture, but only a progressive process of osteoporosis. A total of 392 patients were included in our study, 19 of whom developed refracture after PKP, with an incidence of approximately $4.8 \%$. There are no uniform criteria for the factors affecting refracture after PKP. Therefore, 
adequate assessment to predict the risk of refracture after PKP. Early identification of high-risk patients and intervention can reduce the incidence of refracture and improve patient prognosis.

PKP can be injected into vertebrae by unilateral or bilateral injection. A cement-free section exists between the bone cement and the upper and lower endplate. The cancellous bone in this area is susceptible to collapse under prolonged stress. Therefore, the strength of the vertebrae is positively correlated with the rate of contact between the bone cement and the upper and lower endplate[11]. When the bone cement was injected into the vertebrae with adequate dispersion, the risk of postoperative refracture would reduced. The ideal distribution is when the postoperative bone cement is uniformly diffused over the anterior two-thirds of the vertebrae [15]. Previous meta-analyses have shown no difference in clinical efficacy between unilateral and bilateral injection. By creating a vertebral compression fracture model, Chen [16] concluded that both unilateral and bilateral punctures can significantly increase vertebral stiffness. Unilateral puncture creates a biomechanical imbalance secondary to the uneven diffusion of the bone cement within the vertebrae $[17,18]$. The imbalance of spinal biomechanics is an essential influence on vertebral fractures. When vertebrae ware under prolonged high load, the side with poorer biomechanics is more at risk for refracture. He [19] evaluated the effect of different bone cement distribution on the clinical efficacy by a retrospective analysis. After more than 1 year of follow-up, patients with bilateral punctures are mostly " $\mathrm{H}$ " distributed on both sides of vertebrae, and they can achieve better outcomes in the short term. Currently, many surgeons commonly treat patients using unilateral puncture in their clinical practice [9,20-22]. They concluded that unilateral puncture could achieve acceptable biomechanical stability with cement filling beyond the midline of vertebrae [16]. However, the unilateral puncture approach inevitably fails to effectively diffuse the bone cement into vertebrae due to its limited puncture angle [23]. Therefore, we recommend that bilateral puncture remains the preferred approach when bone cement filling is less effective or when the patient suffers from severe osteoporosis.

Lower BMD reflects the severity of osteoporosis. With decreasing T-values, the incidence of refracture Increases $[6,8-10,12]$. Meanwhile lower BMD means that bone cement is more likely to leak from cancellous bone. The value of BMD was closely related to the course of OVDFs. Qi [24] concluded from examining bone biopsy specimens and blood samples from 206 patients with OVCFs that low BMD is often associated with reduced bone formation capacity, severe loss of vertebral height and large amounts of necrotic bone. Lower BMI often means that patients have a lower long-term protein intake. Protein and amino acids are the raw materials for bone production. When protein is chronically deficient, bone production is reduced, further aggravating the process of osteoporosis.

The more vertebrae fractured in the initial surgery, the greater disruption of the normal spinal biologic force line [25]. Bone cement infusion in multiple segments has a significant impact on the biomechanics and load transfer of the entire spine. Among the bone cement leaks that lead to refractures, intervertebral disc leakage is the most common $[6,13]$. Sun $[26]$ showed that if there is no leakage, the refracture is $7 \%$. However, when disc leakage occurs, this rate increases to $14 \%$. Leakage of bone cement in the 
intervertebral disc accelerates the degeneration of the disc and other structures. The bone cement weakens the mechanical cushioning effect of the disc, resulting in uneven mechanical conduction. In addition, the bone cement can produce mechanical damage to the endplate. Leakage of the bone cement triggers multiple destructive mechanisms that combine to cause refracture.

However, this study has several limitations. First, refracture occurred mostly within 6 months after surgery. However, the long-term follow-up results show that vertebral fractures were inevitable with the progressive course of osteoporosis. In contrast, the mean follow-up time of the study was 12 months, which limits our investigation of the change in the probability of postoperative vertebral refracture over time. Second, our study is a single-center retrospective study. Although internal validation using enhanced Bootsrap can be effective in evaluating model performance, additional large-scale clinical cohorts are still needed for external validation.

\section{Conclusion}

In summary, this study selected four predictors associated with refracture after PKP by LASSO regression. A prognostic model was developed based on these four predictors. The results of internal validation show that this model has good comprehensive performance. The model can well predict the probability of refracture after PKP, which provides a basis for prognosis prediction and personalized treatment plan development for patients with OVCFs.

\section{Declarations}

Acknowledgments This work was supported by grants from the Youth Program of National Natural Science Foundation of China (NO.81801213),Jiangsu Province Postgraduate Research and Practice Innovation Program (SJCX21_1145). We thank all the colleagues for their invaluable assistance during the execution of the present study. We thank all patients and their families.

Data availability The data used and/or analyzed during the current study are available from the corresponding author on reasonable request.

\section{Compliance with ethical standards}

Conflict of interest The authors declare that there is no conflict of interest.

Ethics approval This study was approved by the Institutional Ethics Committee of the Affiliated Hospital of Xuzhou Medical University. This study was performed in accordance with the ethical standards in the 1964 Declaration of Helsinki.

Informed consent Informed consent was obtained from all individual participants included in the study.

Consent to participate Not applicable. 
Consent for publication Not applicable.

\section{References}

1. Lee JK, Jeong HW, Joo IH, et al. Percutaneous balloon kyphoplasty for the treatment of very severe osteoporotic vertebral compression fractures: a case-control study. Spine J. 2018;18(6):962-9. https://doi.org/10.1016/j.spinee.2017.10.006.

2. Zhang H, Xu C, Zhang T, et al. Does Percutaneous Vertebroplasty or Balloon Kyphoplasty for Osteoporotic Vertebral Compression Fractures Increase the Incidence of New Vertebral Fractures? A Meta-Analysis. Pain Physician. 2017;20(1):E13-28.

3. Ko BS, Cho KJ, Park JW. Early Adjacent Vertebral Fractures after Balloon Kyphoplasty for Osteoporotic Vertebral Compression Fractures. Asian Spine J. 2019;13(2):210-5. https://doi.org/10.31616/asj.2018.0224.

4. Zhu JJ, Zhang DS, Lou SL, Yang YH. Surgical treatment of secondary fractures after percutaneous vertebroplasty: A retrospective study. Indian J Orthop. 2019;51(3):269-72. https://doi.org/10.4103/0019-5413.205677.

5. Lavelle WF, Cheney R. Recurrent fracture after vertebral kyphoplasty. Spine J. 2016;6(5):488-93. .https://doi.org/10.1016/j.spinee.2005.10.013.

6. Rho YJ, Choe WJ, Chun YI. Risk factors predicting the new symptomatic vertebral compression fractures after percutaneous vertebroplasty or kyphoplasty. Eur Spine J. 2012;21(5):905 - 11. https://doi.org/10.1007/s00586-011-2099-5.

7. Li YX, Guo DQ, Zhang SC, et al. Risk factor analysis for re-collapse of cemented vertebrae after percutaneous vertebroplasty (PVP) or percutaneous kyphoplasty (PKP). Int Orthop. 2018;42(9):2131-9. https://doi.org/10.1007/s00264-018-3838-6.

8. Gao C, Zong M, Wang WT, Xu L, Cao D, Zou YF. Analysis of risk factors causing short-term cement leakages and long-term complications after percutaneous kyphoplasty for osteoporotic vertebral compression fractures. Acta Radiol. 2018;59(5):577-85. https://doi.org/10.1177/0284185117725368.

9. Wu J, Guan YH, Fan SL. Risk factors of non-surgical vertebral fracture after percutaneous kyphoplasty of single segment thoracolumbar fracture. Zhongguo Gu Shang. 2017;30(9):833-7. https://doi.org/10.3969/j.issn.1003-0034.2017.09.010.

10. Wang YT, Wu XT, Chen $\mathrm{H}$, et al. Adjacent-level symptomatic fracture after percutaneous vertebral augmentation of osteoporotic vertebral compression fracture: a retrospective analysis. J Orthop Sci. 2014;19(6):868 - 76. https://doi.org/10.1007/s00776-014-0610-7.

11. An Z, Chen C, Wang J, et al. Logistic regression analysis on risk factors of augmented vertebra recompression after percutaneous vertebral augmentation. J Orthop Surg Res. 2021;16(1):374. https://doi.org/10.1186/s13018-021-02480-9. 
12. Yang S, Liu Y, Yang H, et al (2016) Risk factors and correlation of secondary adjacent vertebral compression fracture in percutaneous kyphoplasty. Int J Surg. 36(PtA):138142.https://doi.org/10.1016/j.ijsu.2016.10.030.

13. Zhou J, Ma H, Zou D, et al. Correlative factors of secondary fracture after percutaneous kyphoplasty for osteoporotic vertebral compression fracture. Zhongguo Xiu Fu Chong Jian Wai Ke Za Zhi. 2011;25(10):1180-3.

14. Gu YJ, Jiang WY, Yu L, et al. Efficacy of bilateral sagittal cross percutaneous kyphoplasty for preventing recurrent fracture of the cemented vertebrae. Zhongguo Gu Shang. 2019;32(7):630-5. https://doi.org/10.3969/j.issn.1003-0034.2019.07.009.

15. Li Q, Long X, Wang Y, et al. Clinical observation of two bone cement distribution modes after percutaneous vertebroplasty for osteoporotic vertebral compression fractures. BMC Musculoskelet Disord. 2021;22(1):577. https://doi.org/10.1186/s12891-021-04480-6.

16. Chen B, Li Y, Xie D, et al. Comparison of unipedicular and bipedicular kyphoplasty on the stiffness and biomechanical balance of compression fractured vertebrae. Eur Spine J. 2011;20(8):1272-80. .https://doi.org/10.1007/s00586-011-1744-3.

17. Gou Y, Li H, Fu B, Che Z, et al. Short-term effectiveness comparison of unipedicular versus bipedicular percutaneous kyphoplasty for osteoporotic vertebral compression fractures with posterior wall broken. Zhongguo Xiu Fu Chong Jian Wai Ke Za Zhi. 2020;34(10):1281-7. https://doi.org/10.7507/1002-1892.201907001.

18. Tang J, Guo WC, Hu JF, et al. Unilateral and Bilateral Percutaneous Kyphoplasty for Thoracolumbar Osteoporotic Compression Fractures. J Coll Physicians Surg Pak. 2019;29(10):946-50. https://doi.org/10.29271/jcpsp.2019.10.946.

19. He S, Zhang Y, Lv N, et al. The effect of bone cement distribution on clinical efficacy after percutaneous kyphoplasty for osteoporotic vertebral compression fractures. Medicine. 2019;98(50):e18217. https://doi.org/10.1097/MD.0000000000018217.

20. Yin P, Li Z, Zhu S, et al. The treatment of osteoporotic thoraco-lumbar burst fractures by unilateral percutaneous kyphoplasty: A prospective observation study. Eur J Pain. 2020;24(3):659-64. https://doi.org/10.1002/ejp.1516.

21. Tan G, Li F, Zhou D, et al. Unilateral versus bilateral percutaneous balloon kyphoplasty for osteoporotic vertebral compression fractures: A systematic review of overlapping meta-analyses. Medicine (Baltimore). 2018;97(33):e11968. https://doi.org/10.1097/MD.0000000000011968.

22. Li Z, Liu T, Yin P, et al. The therapeutic effects of percutaneous kyphoplasty on osteoporotic vertebral compression fractures with or without intravertebral cleft. Int Orthop. 2019;43(2):359-65. https://doi.org/10.1007/s00264-018-4007-7.

23. Lin J, Qian L, Jiang C, et al. Bone cement distribution is a potential predictor to the reconstructive effects of unilateral percutaneous kyphoplasty in OVCFs: a retrospective study. J Orthop Surg Res. 2018;13(1):140. https://doi.org/10.1186/s13018-018-0839-5. 
24. Qi H, Qi J, Gao J, et al. The Impact of Bone Mineral Density on Bone Metabolism and the Fracture Healing Process in Elderly Chinese Patients With Osteoporotic Vertebral Compression Fractures. J Clin Densitom 2021 Jan-Mar. 2021;24(1):135-45. https://doi.org/10.1016/j.jocd.2020.11.003.

25. Zhai W, Jia Y, Wang J, et al. The clinical effect of percutaneous kyphoplasty for the treatment of multiple osteoporotic vertebral compression fractures and the prevention of new vertebral fractures. Int J Clin Exp Med. 2015;8(8):13473-81.

26. Sun $Y$, Xiong $X$, Wan $D$, et al. Comparison of effectiveness of Vesselplasty and percutaneous kyphoplasty for Kümmell disease. Zhongguo Xiu Fu Chong Jian Wai Ke Za Zhi. 2020;34(12):153944. https://doi.org/10.7507/1002-1892.202007064.

Figures
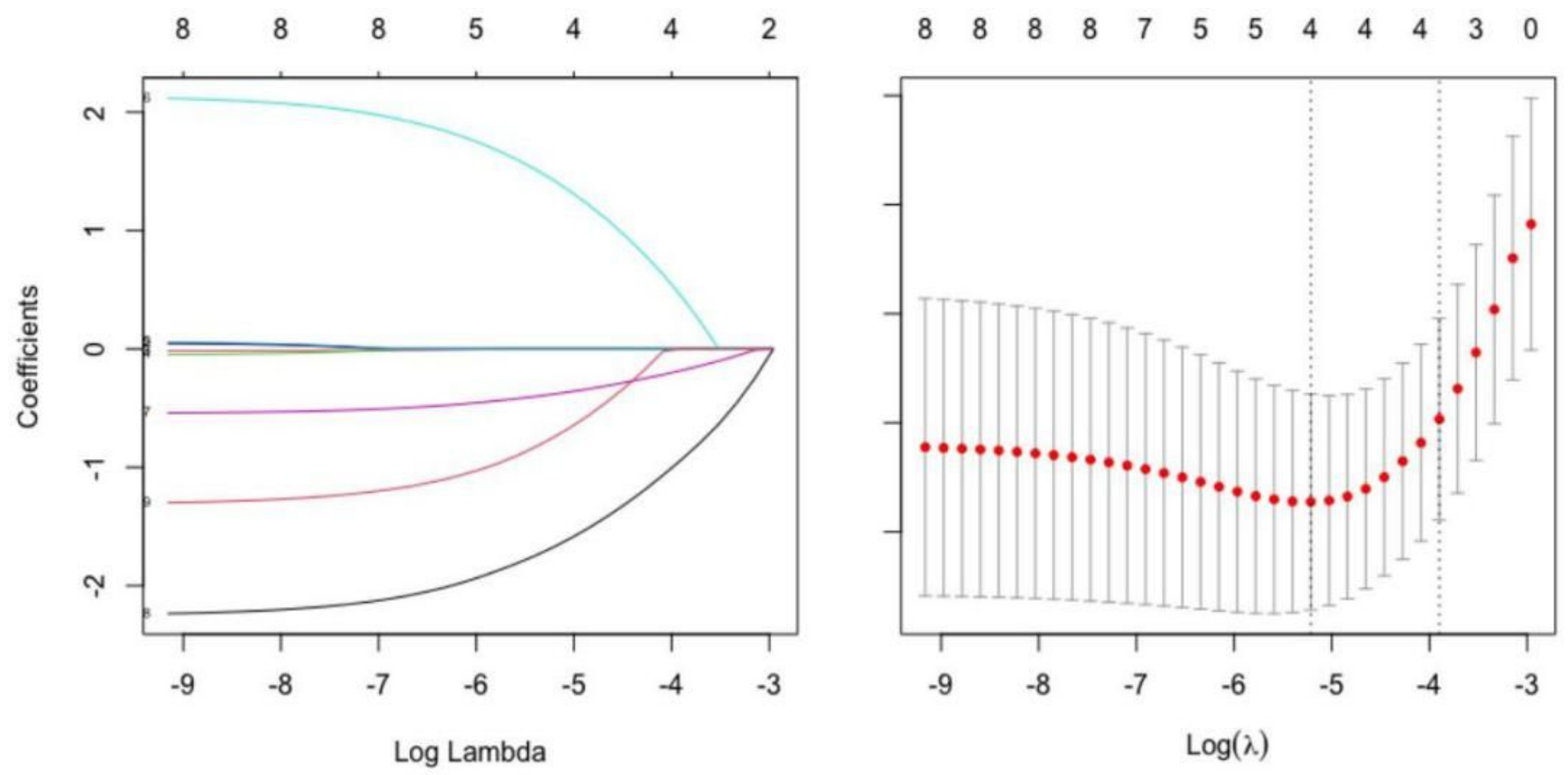

Figure 1

Refracture variable selection by using LASSO regression 
Points

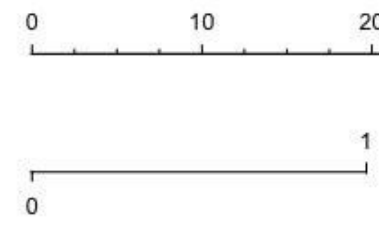

$\mathrm{BMI}$

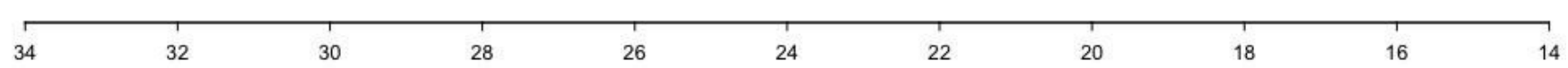

BMD

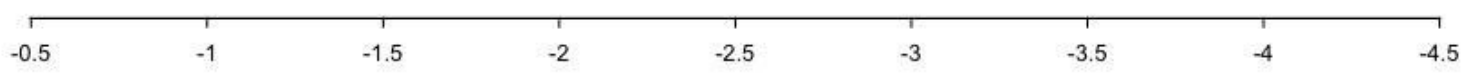

Unilateral

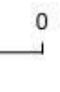

Total Points

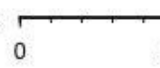

20

40

60

80

100

120

140

160

180

200

Predicted Value

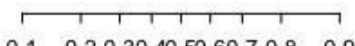

Figure 2

Nomogram predicting refracture after percutaneous kyphoplasty

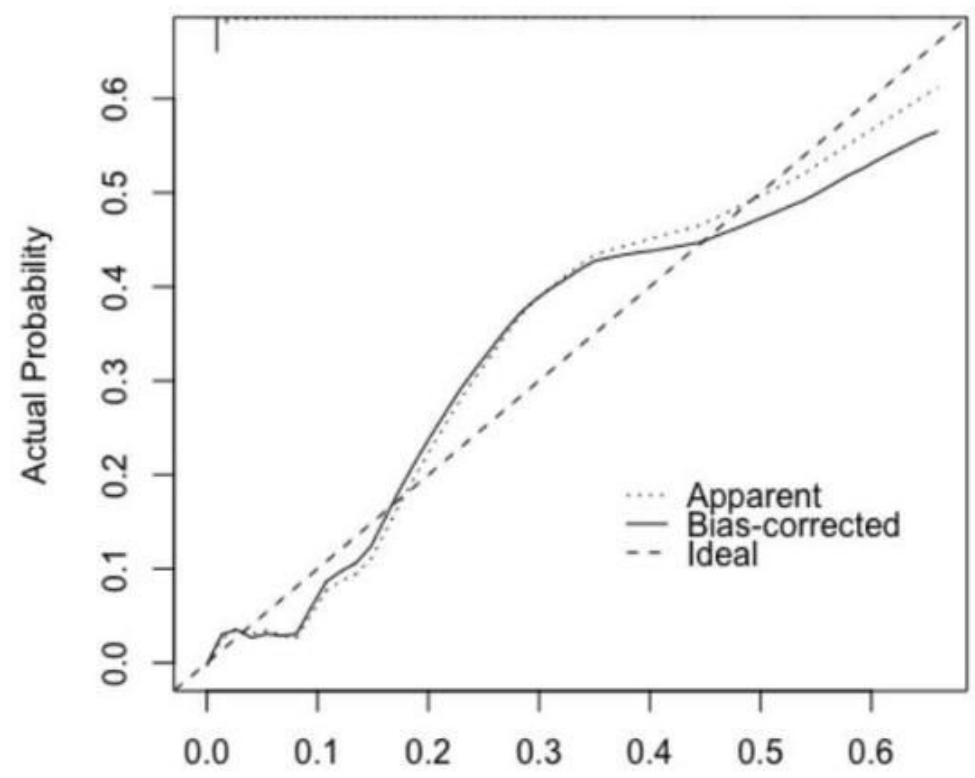

Predicted $\operatorname{Pr}\{$ Fractures $=1\}$ $B=1000$ repetitions, boot

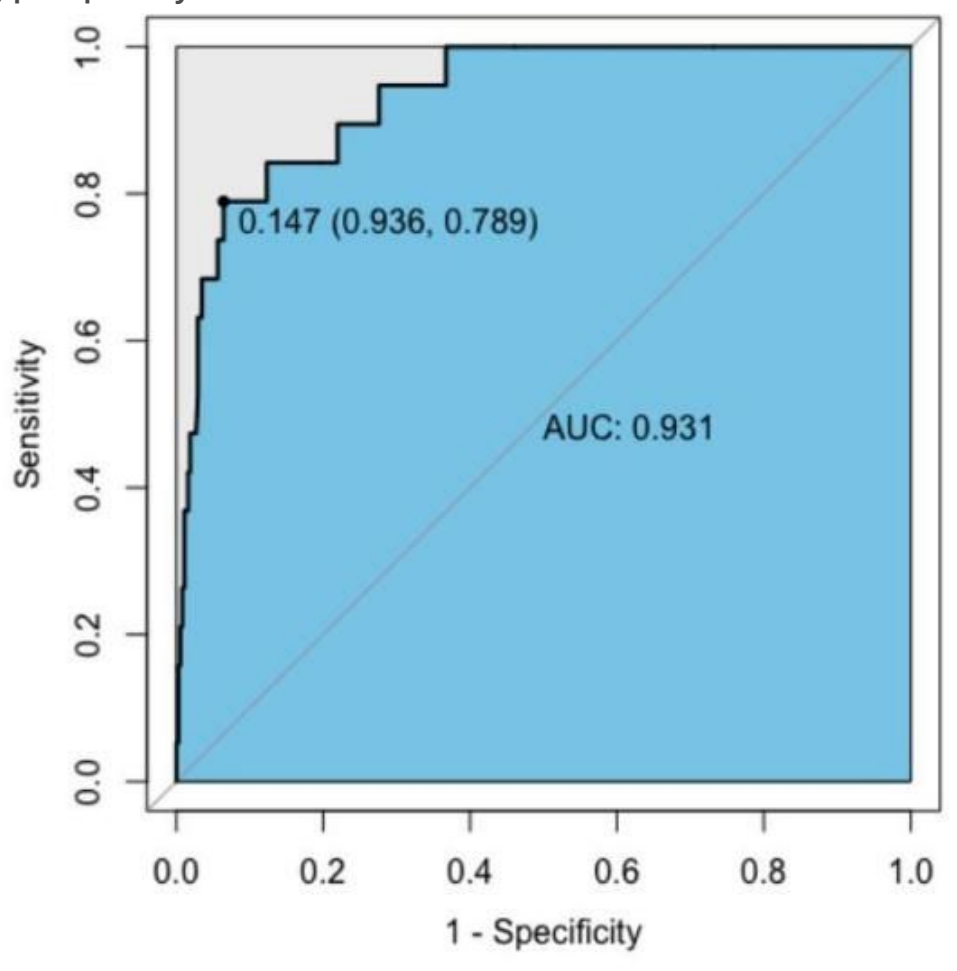

\section{Figure 3}

Calibration plot and ROC curve of the prognostic model

\section{Supplementary Files}

This is a list of supplementary files associated with this preprint. Click to download. 
- TripodChecklist.docx

Page 13/13 